Document downloaded from:

http://hdl.handle.net/10251/123546

This paper must be cited as:

Lazaro, C.; Bessini-Muñoz, JG.; Monleón Cremades, S. (2018). Mechanical models in computational form finding of bending-active structures. International Journal of Space Structures. 33(2):86-97. https://doi.org/10.1177/0266351118794277

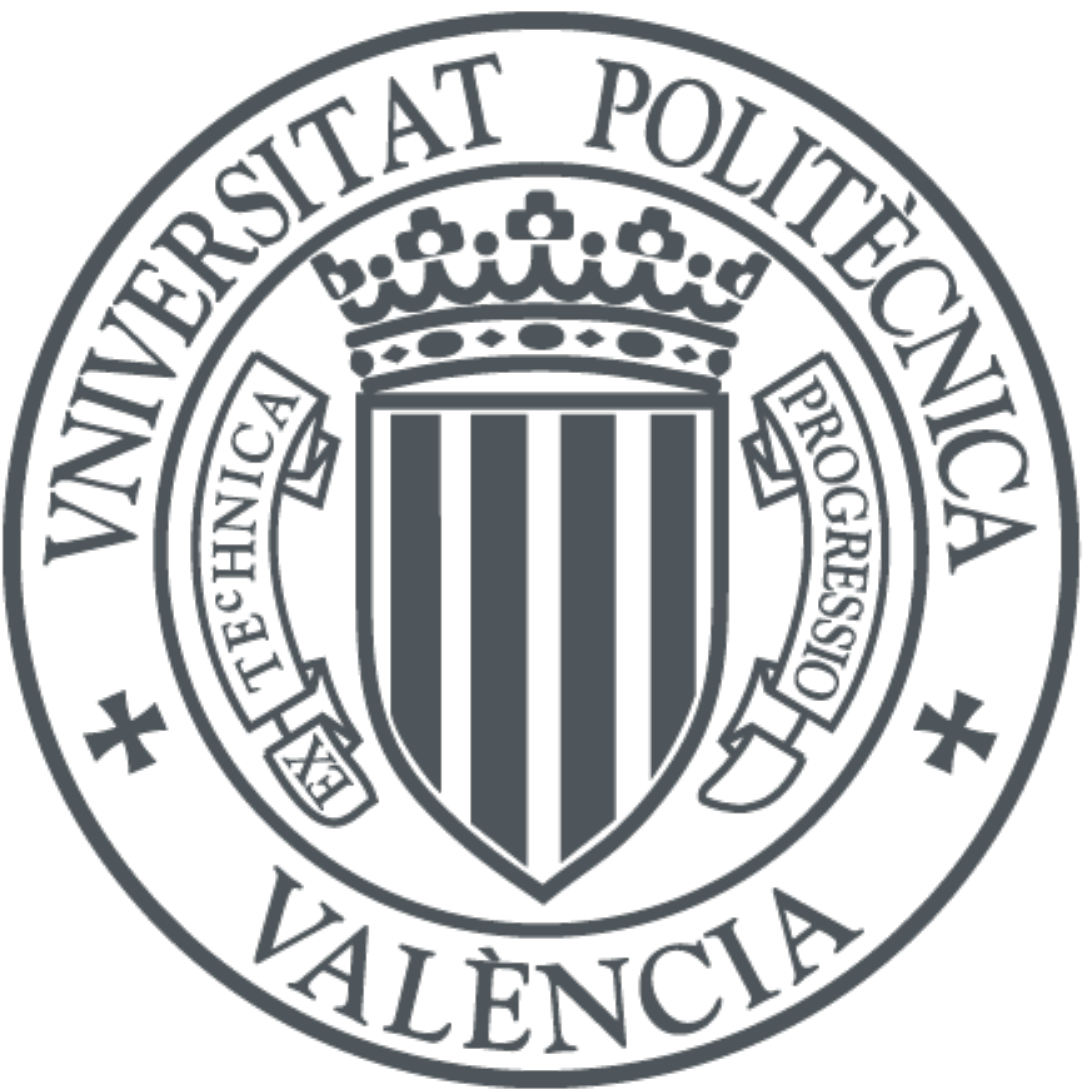

The final publication is available at

https://doi.org/10.1177/0266351118794277

Copyright SAGE Publications

Additional Information 
This paper must be cited as:

Lázaro, C.; Bessini, J.; Monleón, S. (2018). Mechanical models in computational form finding of bending-active structures. International Journal of Space Structures; 33(2): 86-97.

doi: $10.1177 / 0266351118794277$.

The final publication is available at:

https://doi.org/10.1177/0266351118794277

Copyright

SAGE 


\section{Mechanical models in computational form finding of bending-active structures}

Journal Title

$X X(X): 2-26$

(C)The Author(s) 0000

Reprints and permission:

sagepub.co.uk/journalsPermissions.nav

DOI: $10.1177 /$ ToBeAssigned

www.sagepub.com/

SAGE

\section{Carlos Lázaro', Juan Bessini ${ }^{1}$, Salvador Monleón ${ }^{1}$}

\section{Abstract}

This paper reviews the different aspects involved in computational form finding of bending-active structures based on the dynamic relaxation technique. Dynamic relaxation has been applied to form-finding problems of bending-active structures in a number of references. Due to the complex nature of large spatial deformations of flexible beams, the implementation of suitable mechanical beam models in the dynamic relaxation algorithm is a non-trivial task. Type of discretisation and underlying beam theory have been identified as key aspects for numerical implementations. References can be classified into two groups depending on the selected discretisation: finite-difference-like and finite-element-like. The first group includes 3- and 4-degreeof-freedom implementations based on increasingly complex beam models. The second gathers 6-degree-of-freedom discretisations based on co-rotational 3D Kirchhoff-Love beam elements and geometrically exact Reissner-Simo beam elements. After reviewing and comparing implementation details, the advantages and drawbacks of each group have been discussed, and open aspects for future work have been pointed out. 


\section{Keywords}

bending-active structures, computational form finding, Euler-Bernoulli beam theory, Kirchhoff-Love beam theory, dynamic relaxation, finite-difference-like discretisation, finite-element-like discretisation, geometrically exact beam model

\section{Introduction}

Bending-active structures are a kind of structures in which some members are pre-bent and then stabilized to achieve a certain desired configuration. The resulting system may reach considerable stiffness in relation to its weight due to the combination of the shape and slenderness of bent members and the post-bending stabilization by additional means as cables or membranes. The active bending principle has been used in vernacular architecture, for example in the construction of tent-like dwellings (yurts or gers) by Asian nomadic peoples, where flexible timber slats are joined to form a lattice and bent to a cylindrical shaped wall structure and a dome shaped roof.

Since the construction of the Mannheim Multihalle ${ }^{1}$ designed by Frei Otto, which is a pioneering modern application of the active bending principle in architecture, a number of dome shaped grid-shells have been designed and built. Many of them have been designed as temporary or experimental structures as reported by Douthe et $a .^{2}{ }^{2}$ Nicholas et $a .^{3}$, Pone et $a l .{ }^{4}$ or Harding et al. ${ }^{5}$. The basic principle of grid-shells is the use of very flexible members which are joined and bent into a target shape and stabilized by fixing the ground supports in a convenient way. The result is a lightweight shell-like structure which is stiff enough due to its shape, but whose members are light and easy to manipulate during construction. The arrangement of joints and the length and stiffness of members allows for different overall shapes of the dome.

\footnotetext{
${ }^{1}$ Departamento de Mecánica de Medios Continuos y Teoría de Estructuras, Universitat Politècnica de València, Spain

Corresponding author:

Carlos Lázaro, Departamento de Mecánica de Medios Continuos y Teoría de Estructuras, Universitat Politècnica de València, Camino de Vera s/n, 46022 Valencia, Spain

Email: carlafer@mes.upv.es
} 
Bending-active structures are not limited to grid-shells; other realizations include small scale grandstand roofs ${ }^{6}$, umbrellas, and sculptural or ornamental applications. Lienhard et al. ${ }^{7}$ have prepared a comprehensive review of this structural type.

The design process is non-trivial, and involves three stages: (1) determination of the initial shape (form finding); (2) determination of the initial stress state; (3) modelling of the post-stabilization behaviour. Ideally, steps (1) and (2) are carried on simultaneously, as in the case of computational form finding of cable nets. Lienhard ${ }^{8}$ has compared governing variables in form-finding problems of form-active structures with variables in form finding of bending-active structures; he points out that in the former, the mechanical properties of the material are not governing the result, because shape is solely the result of equilibrium. As stated in this reference, '... the form finding of bending-active structures is largely influenced by the length of a beam [...] that is bent as a result of the constraining boundary conditions as well as the mechanical behaviour of the beam or shell elements.' Therefore, the complex nature of the mechanical problem has led in some cases to design processes in which the shape is initially searched using physical models and afterwards the inital state is simulated by means of finite element models, in a so-called 'integral approach' to the design process ${ }^{8}$.

Mainstream computational form-finding techniques rely on Dynamic Relaxation (DR) with different underlying mechanical models. From a numerical point of view, DR can be classified as an explicit method to find an equilibrium configuration. As suggested by D'Amico et al. ${ }^{9}$, explicit methods are well suited to form-finding problems -for which tentative inital configurations may be far from equilibrium,- in contrast with implicit solution methods, widely used in physical simulation of flexible structures under prescribed forces or displacements.

In addition to the pure form-finding problem, the need to solve the mechanical problem appears in the aforementioned steps (2) and (3). The second step may require tracking the full deformation path of the structural elements from an initial unstressed configuration. This usually involves traversing critical points in the equilibrium path, because the target configuration is a post-buckling state of the initial system. After the target shape has been reached and stabilized, the behaviour may not involve large displacements any more (unless the loads are again very 
large,) but it is influenced by the inherited stress state. In this context, four basic features are required for a reliable simulation: (a) a sound mechanical model for flexible members undergoing large displacements of the reference lines and large rotations of the cross sections, and a numerical implementation capable of: (b) traversing critical points in the equilibrium path, (c) allowing the addition and removal (in each stage) of certain members required to bring the system to the desired shape and/or stabilize it, and (d) inheriting the stress state corresponding to a previous stage/configuration. Lienhard ${ }^{8}$ has analysed two commercial finite element software packages and shown some drawbacks regarding features (b), (c) and (d), that lead to disregarding one of them and adapting and completing the other to meet these needs. In this reference, Lienhard proposes a form-finding procedure based on finite element simulations that start from undeformed/unstressed members and drive them to a target configuration by shortening notional elastic cables. This process has been recently improved using an isogeometric finite element implementation (Bauer et al. ${ }^{10}$ ) that allows integration into a CAD environment (Bauer et al. ${ }^{11}$, Längst et al. ${ }^{12}$ ).

Regarding the first mentioned requirement, the mechanical model, numerous references can be found in the context of Finite Element techniques. However, in the applications to bending-active structures most attention has been directed to developing the geometry-related aspects of form finding, and to a lesser extent to the mechanics-related aspects. The following specific issues need be taken into consideration in the mechanical modelling of structures with bending-active members:

1. Large displacements and large rotations of member cross-sections with respect to an initial configuration (generally not in equilibrium in a form-finding process) are to be expected.

2. Because active structures need to behave elastically in the target (bent) configuration -or equivalently, they should be far from yielding,- active members are designed using materials with a high strength-to-Young's modulus ratio and low-depth cross-sections to reach high flexibility and resilience.

3. The previous observation implies that, in spite of displacements and rotations being large, strains will remain small in the target configuration. 
Therefore, beam theories considering linear elastic material will be adequate to reproduce the behaviour of bending-active structures.

The objectives of this work are to provide an overview of the mechanical models that have been used in computational form finding of bendingactive structures, and to review and classify the most relevant references with respect to this point of view. Only references in which explicit methods as dynamic relaxation are used for form finding will be considered, although as it has been mentioned before, other methods have also been used.

\section{Key aspects in the specialization of flexible beam models for dynamic relaxation}

It is assumed that the reader is familiar with the dynamic relaxation (DR) method to determine the equilibrium configuration of a mechanical system (refer, for example, to Barnes ${ }^{13}$ ). The system to be analysed is discretised in a set of nodes. Fictitious masses are associated with model nodes. Stiffness relationships between nodes are defined. The analysis is started from an initial out-of-equilibrium configuration. Internal link forces corresponding to nodal positions at a given instant are calculated using stiffness relationships; external forces acting at nodes can be also introduced. With the resulting residual forces acting on every node, accelerations, velocities and displacements are evaluated. Artificial damping (or suppression of the kinetic energy) is used to bring the system to rest in static equilibrium.

The DR framework has been specialized to form finding of bendingactive structures in different manners. The following aspects are tightly related and define the model specialization:

- Type of discretisation;

- Underlying beam theory.

There are two types of discretisations in DR implementations reported in the literature: finite-difference-like discretisations and finite-elementlike discretisations. In both cases, a key question is the way in which force-configuration relationships between nodes are established. Those relationships are determined in each case by the underlying beam theory, defined by the assumed kinematics, the selected elastic energy terms and the corresponding constitutive relations. 


\section{Beam theories for form finding of bending-active structures}

Computational models for flexible rods rely on a suitable beam theory that models the $3 \mathrm{D}$ deformation of the elastic body using a reduced set of kinematic and static variables. All examined references make use of the classical assumption of cross-sections remaining plane and perpendicular to the deformed centreline of the rod, although with some differences between them. This assumption is a natural choice because strains induced by shear forces, which cause loss of planarity or perpendicularity of cross sections, are negligible in very slender members. The next sections review relevant aspects of the main beam theories in the context of form finding of bending-active structures. They are presented in order of increasing complexity; each theory may be considered a subset of the following one.

\section{Euler-Bernoulli theory}

The first reported solution for planar large deformations of a rod was developed by Euler in 1744, applying calculus of variations to the expression of the bending energy of the rod, and assuming that (a) curvatures are proportional to bending moments -as suggested to him by Bernoulli- and (b) the rod length remains constant; this is equivalent to assuming inextensibility of the rod centreline and neglecting shear deformability. Conjugate variables in Euler-Bernoulli theory are centreline curvatures $\kappa$ (strains) and bending moments $M$ (section forces). The assumed constitutive relation is

$$
M=E I \kappa
$$

and the deformation energy is given by

$$
\mathcal{U}_{E-B}=\frac{1}{2} \int_{L} E I \kappa^{2} d S
$$

Where $d s=d S$ is the centreline length differential in the actual and the reference configuration, and they are equal due to the inextensibility assumption. Axial and shear forces $N, V$ are related to $M$ through equilibrium equations and are not associated with the deformation energy. Configuration variables can be reduced to $x$ and $y$, positions of the centreline points referred to an external fixed frame. Then $d s=$ $\sqrt{1+\left(y^{\prime}\right)^{2}} d x$, with the prime $\left(^{\prime}\right)$ denoting derivatives with respect to $x$. 
The exact expression of the curvature in terms of centreline positions is

$$
\kappa=\frac{1}{R}=\frac{y^{\prime \prime}}{\left(1+\left(y^{\prime}\right)^{2}\right)^{3 / 2}}
$$

The configuration of a flexible rod under compressive loads applied at both ends deduced from this theory is called elastica. The linearised version of the curvature, $\kappa=y^{\prime \prime}=v^{\prime \prime}$, with $v$ denoting the beam transverse displacement, is used to formulate the linear planar bending theory of beams.

A refinement of Euler-Bernoulli theory can be obtained relaxing the inextensibility assumption. It involves the elongation energy

$$
\frac{1}{2} \int_{L} E A \epsilon^{2} d S
$$

together with the constitutive relation between axial force and centreline strain,

$$
N=E A \epsilon
$$

and the definition of the axial strain

$$
\epsilon=\frac{d s}{d S}-1
$$

Non-linear solutions derived with this theory are called extensible elasticas. For the linear beam theory, equation (6) reduces to $\epsilon=u^{\prime}$.

\section{Kirchhoff-Love theory}

In 1859 Kirchhoff $^{14}$ proposed a 3D theory for very slender rods with extensible centreline that was developed by Love $(1927)^{15}$. KirchhoffLove theory leads to negligible shear deformations, keeps Bernoulli's moment-to-curvature proportionality in both principal axes, and models torsion according to Saint-Venant's theory (Dill ${ }^{16}$ ); it has been extensively used for developing finite elements for beams in the realm of small or moderately large displacements and rotations. Conjugate variables can be defined with respect to a set of orthogonal axes $\{1,2,3\}$ located at each point of the deformed centreline, with axis 1 being tangent to the centreline, and 2,3 coincident with the cross-section principal axes: centreline axial strains $\epsilon$, torsional strains $\kappa_{1}$ and curvatures $\kappa_{2}, \kappa_{3}$ are 
conjugate to axial forces $N$, torsional moments $M_{1}$ and bending moments $M_{2}, M_{3}$, respectively. Shear forces $V_{2}, V_{3}$ do not deform the rod and are related to previous variables through equilibrium relations. Constitutive equations are

$$
N=E A \epsilon \quad M_{1}=G J \kappa_{1} \quad M_{2}=E I_{2} \kappa_{2} \quad M_{3}=E I_{3} \kappa_{3}
$$

and the deformation energy is

$$
\mathcal{U}_{K-L}=\frac{1}{2} \int_{L}\left(E A \epsilon^{2}+G J \kappa_{1}^{2}+E I_{2} \kappa_{2}^{2}+E I_{3} \kappa_{3}^{2}\right) d S
$$

Configuration variables are: positions of centreline points referred to a fixed frame, $x, y, z$, and rotational parameters needed to describe cross-sectional rotations. Several choices can be made in this respect, and an adequate selection of sectional reference frames (using torsion free Bishop ${ }^{17}$ frames) allows the use of only one rotational parameter describing the section torsional rotation $\theta_{1}$, because cross sections remain perpendicular to centreline tangents. Thus, $\kappa_{1}=d \theta_{1} / d S$, and the curvatures $\kappa_{2}$ and $\kappa_{3}$ can be expressed through differential-geometric relations in terms of centreline positions and their derivatives. The derivation of linear theories based on Kirchhoff's kinematics is much simpler because the curvatures reduce to $\kappa_{2}=v^{\prime \prime}$ and $\kappa_{3}=w^{\prime \prime}$, with $v$ and $w$ denoting transverse displacements in the beam. In finite element implementations it is usually convenient to keep the (small) rotational degrees of freedom $\theta_{2}$ and $\theta_{3}$ and use the linearised constraints $\theta_{2}=v^{\prime}$ and $\theta_{3}=w^{\prime}$. Then, curvatures are simply the first derivatives of the flexural rotations.

If the rod is considered inextensible, axial forces become a consequence of equilibrium, and Kirchoff-Love theory specializes into the threedimensional version of Euler-Bernoulli theory.

\section{Other theories}

Reissner ${ }^{18}$ proposed a 3D theory in which cross-section rotations are independent from centreline tangents. In this theory, kinematics is based on Timoshenko's assumption for shear-deformable beams -originally introduced for small displacements and deformations- according to which cross-sections remain plane but not necessarily perpendicular to the centreline. In Reissner's theory, as in both previously described ones, 
displacements and rotations may be arbitrarily large. In addition to the previous constitutive equations (7), equations relating shear forces to shear strains $\gamma_{2}, \gamma_{3}$ have to be introduced

$$
V_{2}=G A_{v 2} \gamma_{2} \quad V_{3}=G A_{v 3} \gamma_{3}
$$

and additional terms for shear deformation energies need also be added to equation (8):

$$
\frac{1}{2} \int_{L}\left(G A_{v 2} \gamma_{2}^{2}+G A_{v 3} \gamma_{3}^{2}\right) d S
$$

In this case, centreline positions and sectional (finite) rotations are used as configuration variables. This theory has been successfully developed by Simo ${ }^{19}$ and other authors for the simulation of flexible beams in the context of finite elements, using finite rotations to model the behaviour of cross-sections. The adjective geometrically exact has been used to refer to these models.

To put these theoretical developements into perspective, it is necessary to mention the more general Cosserat rod theory, that models the rod as a space curve with three vectors attached to each point. The first is the tangent vector to the centreline curve; the other two are called directors and characterize the configuration of material fibres of the cross-section. Cosserat rods were developed by Green, Naghdi and Wenner ${ }^{20}$ on the basis of Cosserats' ${ }^{21}$ theory for directed continua.

Both Reissner-Simo and Kirchhoff-Love theories can be classified as special cases of Cosserat rods: in the first case, directors are chosen orhtonormal in the undeformed state, and are constrained to remain orthonormal during the deformation; in the second (Kirchhoff-Love), orthogonality to the tangent vector is also enforced.

More complex theories considering out-of-plane deformation modes of cross-sections have been developed by other authors (as $\mathrm{Simo}^{22}$ or Hodges $^{23}$ ) although their increased number of variables makes them more difficult to apply to shape finding of bending-active structures.

\section{Types of discretisation in DR procedures for flexible rods}

Two groups of discretisations can be found in the literature: (i) finitedifference-like discretisations using 3 or 4 degrees of freedom per node, and (ii) finite-element-like methods with 6 DoFs per node. The main 
distinction is found in the way strain measures and internal forces exerted to model nodes are evaluated.

In finite difference discretisations, discrete strain measures at nodes are deduced from difference schemes between nodal degrees of freedom using groups of two, three or four nodes. Internal forces acting on nodes may be calculated either (a) using the discrete strain measures (assuming linear constitutive behaviour) and ad hoc equilibrium relations; or (b) deriving expressions of the internal forces from energy methods and discretising these expressions by means of finite-difference schemes.

In the case of finite-element-like discretisations, the link between nodes is considered as a beam finite element. Relations between element end-forces and relative displacements/rotations of end-nodes are derived making use of beam theory and energy methods. In the following sections we review the main contributions in each group.

\section{Finite-difference discretisations}

In this group we are placing in chronological order the contributions by Adriaenssens \& Barnes ${ }^{24}$; Barnes, Adriaenssens \& Krupka ${ }^{25}$; Du Peloux, Tayeb, Lefevre, Baverel \& Caron ${ }^{26}$; and D'Amico, Zhang \& Kermani ${ }^{9}$.

The first reference (2001) is based on the Euler-Bernoulli theory with the extensibility assumption. In a certain configuration, axial strains $\epsilon$ are calculated from nodal coordinate differences, and link forces in the direction of lines joining nodes are obtained using the constitutive equation (5) for axial forces. In order to account for bending effects, groups of three nodes are considered: the bending moment at node $j$ is got using a discrete measure of the curvature of each three-node group instead of equation (3)

$$
\kappa_{j}=2 \frac{\sin \alpha_{j}}{d_{i k}}
$$

and the constitutive equation (1) $M_{j}=E I \kappa_{j}$ (see Figure 1). The contribution of the three-node group to in-plane shear forces acting on each node is deduced from equilibrium, so that end forces produce a moment $M_{j}$ on the middle $j$ node.

$$
S_{i j}=\frac{M_{j}}{d_{i j}} \quad S_{j k}=\frac{M_{j}}{d_{j k}}
$$

Adriaenssens \& Barnes prove that, for isotropic cross-sections (sections 


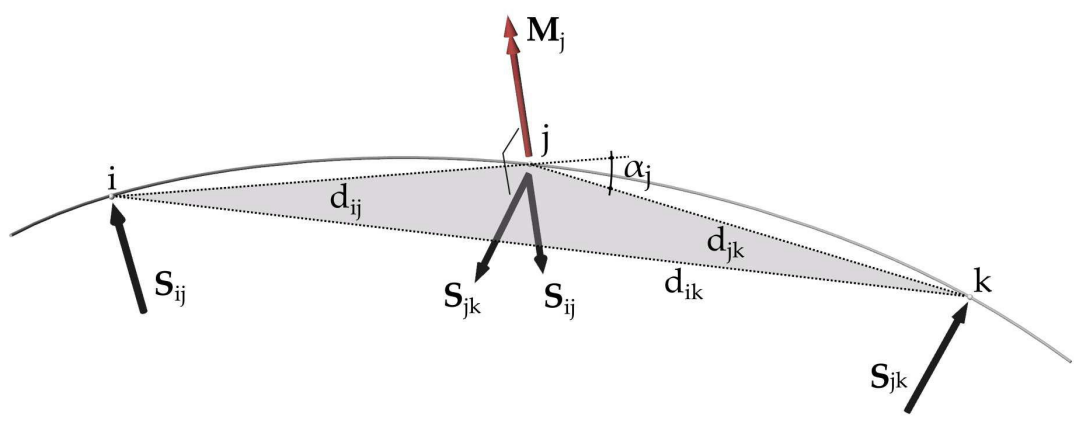

Figure 1. Discretisation in Adriaenssens \& Barnes ${ }^{24}$

with uniform second moment of area), torsional moments are related to bending moments through an equilibrium equation and no additional forces due to torsion are needed for the dynamic relaxation procedure. Thus, shear forces are vector-added to link (axial) forces in order to compute residual forces acting on each node, and the dynamic relaxation procedure can be performed. In this way, only three degrees of freedom per node are needed, and the simplest beam theory is used in the process, with the counterpart of the limitation imposed to cross-sections, which need be square or circular shaped (full or hollow.)

The second reference ${ }^{25}$ (2013) accounts for the case of non-uniform cross sections: in addition to considering the effect of bending through three-node in-plane shear forces as in the previous reference, out-ofplane shear forces acting on four-node groups are calculated using the constitutive equation for torsion (7) $M_{j k 1}=G J \kappa_{j k 1}$ and a discrete measure of the torsional strain $\kappa_{1}$ obtained from the nodal coordinates of the four-node group (see Figure 2).

$$
\kappa_{j k 1}=\frac{\phi-\phi_{0}}{d_{j k}}
$$

where $\phi_{0}$ is the angle formed by planes $i j k$ and $j k l$ at the start of the process. Out of plane forces $P_{i j}$ and $P_{j k}$ are determined to be in equilibrium with $M_{j k 1}$. With this set-up, in-plane as well as out-ofplane forces are added to axial forces to calculate residuals for DR. The underlying theory is a simplified version of Kirchhoff-Love theory, in 


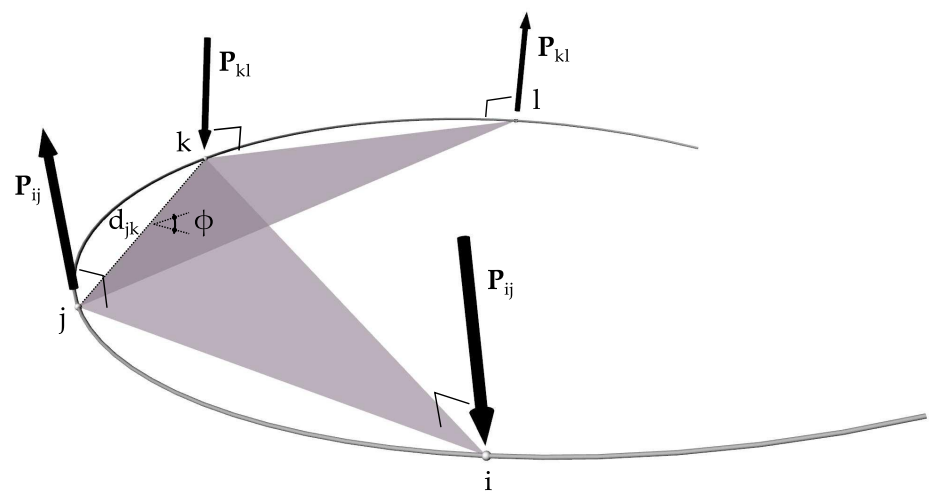

Figure 2. Discretisation in Barnes et al. ${ }^{25}$

which (apart from the constitutive equations for extension and torsion) only one bending constitutive equation is entering the formulation (inplane bending). This simplifying assumption has two consequences: on one side, the orientation of one principal bending axis at a node is forcefully contained into the plane formed by the node itself and the two adjacent ones; on the other, out-of-plane bending stiffness has to be indirectly considered through a factor that modifies the torsional stiffness $G J$. This discretisation keeps the advantage of working with only three degrees of freedom in the DR process.

The method proposed in the third reference (2015) by Du Peloux et $a l .{ }^{26}$ is close to recent developments in the field of computer animation (Bertails et al. ${ }^{27}$, Bergou et al. ${ }^{28}$ ) which relate the geometry of discrete curves (an ordered set of nodes) to their mechanical behaviour using a finite difference scheme. In this reference, the minimal set of degrees of freedom per node that keeps full consistency with Kirchhoff-Love theory is used: three degrees of freedom for nodal positions and one rotational degree of freedom to keep track of torsion. The latter measures the angular difference between cross-sectional material (principal) frames and torsion-free (Bishop) frames. A discrete measure of oriented curvatures using the coordinates of three consecutive nodes is employed. Torsional strains are calculated as finite differences between nodal torsional rotations. In order to calculate nodal forces and twisting moments, Du Peloux et al. discretise the expressions of the internal forces and torsional 


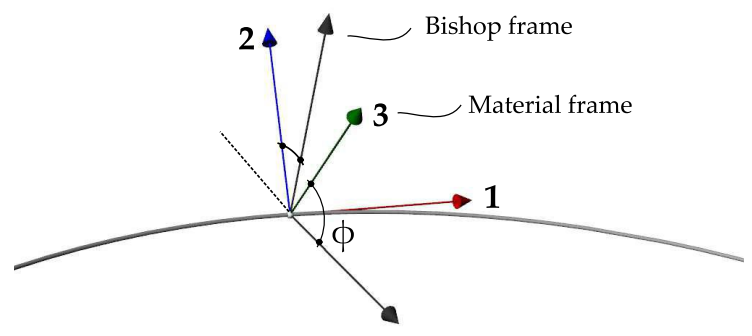

Figure 3. Discretisation in Du Peloux et al. ${ }^{26}$

moment in the rod, obtained as derivatives of the elastic deformation energy with respect to positions and torsional rotation. A simplifying assumption for the evaluation of the derivatives is that torsional waves propagate in an instantaneous manner compared to bending waves. The discretisation of internal forces and twisting moments to obtain forces and moments acting on nodes follows a finite-difference scheme. This computation depends on the torsional stiffness $G J$, and the bending stiffness $\mathrm{EI}_{2}$ and $\mathrm{EI}_{3}$. The rod is considered therefore inextensible. Force and moment residuals are calculated at each node for performing DR. Inextensibility is enforced by means of penalty forces at nodes.

The last reference (2016) by D'Amico et al. ${ }^{9}$ makes also use of a discrete approach to 3D curved rods as Du Peloux et al.. It can be considered an improvement of Barnes et al. because it keeps track of the angular difference between material frames and Bishop frames in case that torsional constraints are imposed at the ends of the rod. The orientation of tangent vectors at nodes is found using Catmull-Rom interpolation; the other two vectors of the Bishop frame are found by parallel transport and then rotated to obtain the material frame. However, in contrast to Du Peloux et al., these rotation angles are not considered degrees of freedom of the system, but initially given data. Curvatures and torsional strains are calculated operating with coordinates and frame orientations at a given node and the immediately adjacent ones (three-node groups). The total moment at a node is the result of vector-adding the components given by constitutive equations (7). Nodal shears acting on every node of a group of three are calculated imposing equilibrium with the total moment at the mid node; they are vector-added to forces from subsequent groups and to 
Table 1. Comparison between references using finite-difference-like discretisations

\begin{tabular}{|c|c|c|c|}
\hline reference & no. of DoFs & beam theory & stiffness parameters \\
\hline Adriaenssens et al. ${ }^{24}$ & 3 & extensible E-B & $E A, E I$ \\
\hline Barnes et al. ${ }^{25}$ & 3 & modified extensible K-L & $E A, E I, G J$ \\
\hline Du Peloux et al. ${ }^{26}$ & 4 & inextensible K-L & $G J, E I_{2}, E I_{3}$ \\
\hline D'Amico et al. ${ }^{9}$ & 3 & extensible K-L & $E A, G J, E I_{2}, E I_{3}$ \\
\hline
\end{tabular}

the axial link forces at nodes in a similar fashion as in Barnes et al. to calculate residuals for DR. This set-up makes therefore use of KirchhoffLove theory but forces the orientation of the principal axes at a node to keep a fixed angle to the corresponding three-node plane due to the restricted number of degrees of freedom.

Table 1 includes a summary of the main features of finite-differencelike parametrisations. Moreover, all references use equilibrium relations to obtain nodal forces for the DR process, excepting the one by Du Peloux et al. that evaluates internal forces as derivatives of the deformation energy with respect to kinematic variables.

\section{Finite-element discretisations}

The following references have been included in this category: Li \& Knippers ${ }^{29}$; D’Amico, Kermani \& Zhang ${ }^{30}$; D’Amico, Kermani, Zhang, Shepherd \& Williams ${ }^{31}$; and Senatore \& Piker $^{32}$. They base on previous work by Williams thoroughly explained in Adriaenssens ${ }^{33}$. All of them share a common implementation feature that allows their classification into a well-established formulation for flexible rod finite elements: the co-rotational formulation. The rod is discretised into elements that can undergo large rotations, but at local level use small-displacement/rotation relationships. We have also included the reference by Bessini, Lázaro \& Monleón ${ }^{34}$ that implements Reissner-Simo model, which is capable to deal with large rotations of cross-sections with no limitation to their relative magnitude at the element level.

The proper numerical treatment of large rotations is a main concern in the computational solution of this type of problems. This fact was readily recognized by Argyris ${ }^{35}$ to which the reader is referred for a detailed account of the following ideas. From a mathematical point of view, a rotation is an element of the special orthogonal group $\mathrm{SO}(3)$. The elements of this non-additive and non-commutative group can be 
numerically represented in several manners. Before reviewing the corotational formulation, the following section summarizes (in a nonexhaustive manner) the most important alternative parametrizations for rotations.

Parametrization of finite rotations Rotations, as members of the special orthogonal group, can be represented by means of $3 \times 3$ orthogonal matrices $\Lambda$ of determinant 1 . The columns of the rotation matrix are the components of the rotated frame. The main drawback of this representation is the high number of required parameters: 9 nonindependent scalars related by 6 conditions.

Euler's theorem establishes the equivalence between the matrix representation of rotations and the pseudo-vectorial representation $\boldsymbol{\theta}$ : the modulus $\theta$ of $\boldsymbol{\theta}$ represents the rotation angle and its orientation determines the rotation axis. The relationship between $\Lambda$ and $\boldsymbol{\theta}$ is given by Rodrigues formula ${ }^{36}$ :

$$
\boldsymbol{\Lambda}=\cos \theta \mathbf{1}+\frac{\sin \theta}{\theta} \widehat{\boldsymbol{\theta}}+\frac{1-\cos \theta}{\theta^{2}} \boldsymbol{\theta} \otimes \boldsymbol{\theta}
$$

where $\widehat{\boldsymbol{\theta}}$ is the skew-symmetric matrix with axial vector $\boldsymbol{\theta}$. This representation uses a minimum number of parameters and the correspondence with $\Lambda$ is one-to-one up to $\theta=\pi$, but it has the drawback that addition of pseudo-vectors does not represent composition of rotations (hence the pseudo- prefix). However, it has been successfully employed to formulate finite elements for large rotation problems.

An alternative pseudo-vectorial 3-parametrisation consists in using $\psi=$ $2 \tan (\theta / 2) \mathbf{e}$, with $\theta$ equal to the rotation angle and $\mathbf{e}$ being a unit vector in the direction of the rotation axis. The relation between both pseudo-vectors is $\boldsymbol{\psi}=\frac{2 \tan (\theta / 2)}{\theta} \boldsymbol{\theta}$. The components of $\boldsymbol{\psi}$ are sometimes called Rodrigues parameters (Crisfield ${ }^{37}$ ). Rodrigues formula transforms through substitution into an expression with no trigonometric functions

$$
\boldsymbol{\Lambda}=\mathbf{1}+\frac{1}{1+\frac{1}{4} \boldsymbol{\psi}^{\top} \boldsymbol{\psi}}\left(\widehat{\boldsymbol{\psi}}+\frac{1}{2} \widehat{\boldsymbol{\psi}}^{2}\right)
$$

This alternative pseudo-vector has been partially used by Williams (Adriaenssens ${ }^{33}$ ) and by D'Amico et al. ${ }^{30,31}$ (we will expand on this in the next section).

Other classical 3-parameter representations, as Euler's angles or Cardano's angles are not advantageous compared to pseudo-vector 
representations, and therefore haven't found use in mechanical modelling of slender beams.

An alternative system to represent rotations is the use of unit quaternions. They are 4-tuples of real numbers $\left(q_{0}, q_{1}, q_{2}, q_{3}\right)$ related by the equation $q_{0}^{2}+q_{1}^{2}+q_{2}^{2}+q_{3}^{2}=1$. The three later quantities can be interpreted as a vector $\mathbf{q}$. The relationship to the rotation matrix is

$$
\boldsymbol{\Lambda}=\left(q_{0}^{2}-1\right) \mathbf{1}+2 q_{0} \widehat{\mathbf{q}}+2 \mathbf{q} \otimes \mathbf{q}
$$

Unit quaternions and rotation pseudo-vectors are related as follows

$$
\left(q_{0}, \mathbf{q}\right)=\left(\cos \frac{\theta}{2}, \sin \frac{\theta}{2} \mathbf{n}\right) \quad \text { with } \quad \mathbf{n}=\frac{\boldsymbol{\theta}}{\theta}
$$

Quaternions are endowed with an algebra that provides an efficient tool to operate with rotations; this fact and the limited number of parameters compared to rotation matrices makes unit quaternions a preferred choice to store and keep track of rotations.

Argyris $^{35}$ showed that the exponential operator acting on a skewsymmetric matrix, $\exp (\widehat{\mathbf{a}})=\sum_{1}^{\infty} \frac{1}{n !} \widehat{\mathbf{a}}^{n}$, results in an orthogonal matrix that can be calculated using Rodrigues formula (14). Considering the group character of rotations, this observation leads to the introduction of a local parametrization through spins $\Delta \boldsymbol{\theta}$, also called local rotational parameters: a new rotation $\Lambda^{(t+1)}$ close to the original one $\Lambda^{(t)}$ is got through the formula

$$
\boldsymbol{\Lambda}^{(t+1)}=\exp (\widehat{\Delta \boldsymbol{\theta}}) \boldsymbol{\Lambda}^{(t)}
$$

Spins have been used as rotational degrees of freedom for finite element implementations based on Reissner's beam theory $\left(\mathrm{Simo}^{19}\right)$. They have been also used to update nodal frames in the finite-element-based discretisations that will be reviewed in the next section.

Discretisations based on the co-rotational formulation The corotational technique was initially proposed in the 1970s by Wempner and by Belytschko et al. (Crisfield ${ }^{38}$, which includes a review of the history and the essentials of this method.)

The discretisation associated to the co-rotational formulation splits the rod into 2-node elements; at a given instant, the configuration is defined by nodal positions and nodal frames. The large-displacement/rotation mechanical problem is divided into two sub-problems: (a) modelling the 


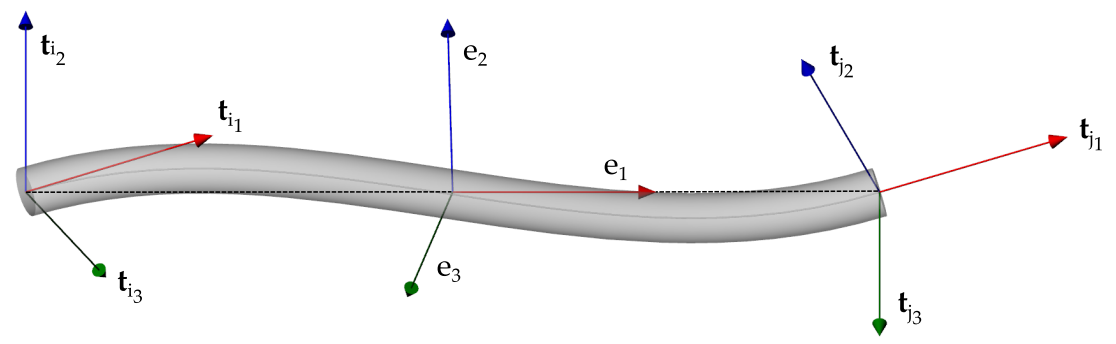

Figure 4. Co-rotational set-up

mechanical behaviour of finite elements between nodes in terms of local displacements and rotations, and relating the latter to the changes in positions and orientations of each node; (b) keeping track of changes in nodal positions and nodal frames, with no restriction in their magnitude.

The first sub-problem requires to define and keep track of element frames, in order to quantify angular differences with nodal frames (Figure 4). If the discretisation is sufficiently refined, these differences will be small, and the element behaviour can be even modelled with a linear beam theory, or considering moderately large displacements and small rotations. Nodal unit base vectors $\mathbf{t}_{i \alpha}, \mathbf{t}_{j \alpha}$ and element base vectors $\mathbf{e}_{\alpha}$ allow to approximate the (small) nodal rotations at some instant as (Crisfield $^{37}$ )

$$
\begin{aligned}
\varphi_{i 1} & =\frac{1}{2}\left(\mathbf{t}_{i 3}^{\top} \mathbf{e}_{2}-\mathbf{t}_{i 2}^{\top} \mathbf{e}_{3}\right) \\
\varphi_{i 2} & =\frac{1}{2}\left(\mathbf{t}_{i 1}^{\top} \mathbf{e}_{3}-\mathbf{t}_{i 3}^{\top} \mathbf{e}_{1}\right) \\
\varphi_{i 3} & =\frac{1}{2}\left(\mathbf{t}_{i 2}^{\top} \mathbf{e}_{1}-\mathbf{t}_{i 1}^{\top} \mathbf{e}_{2}\right) \\
\varphi_{j 1} & =\frac{1}{2}\left(\mathbf{t}_{j 3}^{\top} \mathbf{e}_{2}-\mathbf{t}_{j 2}^{\top} \mathbf{e}_{3}\right) \\
\varphi_{j 2} & =\frac{1}{2}\left(\mathbf{t}_{j 1}^{\top} \mathbf{e}_{3}-\mathbf{t}_{j 3}^{\top} \mathbf{e}_{1}\right) \\
\varphi_{j 3} & =\frac{1}{2}\left(\mathbf{t}_{j 2}^{\top} \mathbf{e}_{1}-\mathbf{t}_{j 1}^{\top} \mathbf{e}_{2}\right)
\end{aligned}
$$

For explicit problems, once nodal rotations are known, linear beam relations between forces, moments, displacements and rotations in a beam 
can be used to get the applied moments and forces at the ends of each element. Alternatively, axial force dependent non-linear terms could also be used to obtain end forces and moments. Force and moment residuals $\mathbf{f}_{i}, \mathbf{m}_{i}$ acting on node $i$ are subsequently evaluated, and from them, linear and angular accelerations $\mathbf{a}_{i}, \boldsymbol{\alpha}_{i}$ follow from D'Alembert's principle

$$
\begin{aligned}
\mathbf{f}_{i} & =m_{i} \mathbf{a}_{i} \\
\mathbf{m}_{i} & =\mathbb{I}_{i} \boldsymbol{\alpha}_{i}+\boldsymbol{\omega}_{i} \times \mathbb{I}_{i} \boldsymbol{\omega}_{i}
\end{aligned}
$$

Because there is no need to exactly reproduce the dynamics in the DR process, an isotropic inertia tensor may be chosen in order to simplify equation (20b):

$$
\mathbf{m}_{i}=\mathbb{I}_{i} \boldsymbol{\alpha}_{i}
$$

With the selected time integration technique, nodal displacements $\Delta \mathbf{x}_{i}$ and local rotation increments (spins) $\Delta \boldsymbol{\theta}_{i}$ corresponding to the time step $t \rightarrow t+1$ are calculated.

The second sub-problem involves updating large rotations of nodal and element frames from an given configuration to the next. In the case of nodal frames, the multiplicative update from equation (18) together with Rodrigues formula (14) to evaluate the exponential are used:

$$
\mathbf{t}_{i \alpha}^{(t+1)}=\exp \left(\widehat{\Delta \boldsymbol{\theta}}_{i}\right) \mathbf{t}_{i \alpha}^{(t)}
$$

for node $i$ and $\alpha=1,2,3$. For element frames, the unit vector $\mathbf{e}_{1}^{(t+1)}$ is computed in a straightforward way from the updated element nodal positions. However, updating the other two vectors requires an interpolation between previously evaluated element nodal frames. Two approaches to this problem are described in Crisfield ${ }^{37}$.

All reviewed references in this group use the co-rotational framework, with some differences in the rotation updating schemes and the mechanical model for the beam element.

A simplification, common to all references, is to avoid the introduction of full element frames: only $\mathbf{e}_{1}$ is computed (as the normalised difference 